\title{
Microscopic Studies of Irradiated Wood by UV light
}

\author{
Petr Svora ${ }^{1,2,3^{*}}$, Sylwia Svorová Pawelkowicz ${ }^{1}$ and Zdeněk Prošek ${ }^{3}$ \\ 1. Centre of Instrumental Techniques, Institute of Inorganic Chemistry, AS CR, v.v.i., Husinec-Rez, \\ Czech Republic \\ 2. Department of Material Analysis, Institute of Physics, AS CR, v.v.i., Prague, Czech Republic \\ 3. University Centre for Energy Efficient Buildings, Czech Technical University in Prague, Buštěhrad, \\ Czech Republic \\ * Corresponding author: svora@fzu.cz
}

The wood itself is not resistant to the sunlight, more precisely, to the UV radiation. The wood matter is a mixture of wide range of organic compounds, and the main components are lignin and cellulose. The UV-light induced degradation of these two chemical substances is responsible for the color change of wood. The goal of the study is to describe changes occurring on microscopic scale in wood subjected to the UV irradiation. For this purpose, high resolution scanning microscopy (SEM/HRSEM) and transmission electron microscopy (TEM) was used [1,2]. This work is a part of a larger project focused on interaction between the wood and $\mathrm{TiO}_{2}$ planar particles under the UV radiation. Our hypothesis is that planar particles of $\mathrm{TiO}_{2}$ are capable of reducing the negative influence of UV light on wood.

For this study, we have chosen pine wood (as a representative of conifer tree) and beech wood (as a representative of broad leaves tree), as both are easily degradable by UV light. Irradiated and reference samples were inspected with SEM and TEM. Proper sample preparation played an important role in obtaining the required information. Samples for HRSEM were prepared in the same way as metallographic samples. They were embedded in epoxy resin, evacuated and after setting grinded and partially polished on a mechanical grinder-polisher using silicon carbide waterproof abrasive paper of successive grits: 500 (wet process with water as lubricant); 1200, 2000, 4000 (dry process) - each step of 3 minutes duration. The final polishing was done with diamond pastes $(3 \mu \mathrm{m}, 1 \mu \mathrm{m}$, MD dur plate) each step of 5 minutes duration. A single specimen force applied was $5 \mathrm{~N}$, rotation speed of the head $50 \mathrm{rpm}$, rotation speed of the disc $40 \mathrm{rpm}$. The rotation of the head and disc was set counter-clockwise. For the final polishing also a precision ion polishing system (PECS) was used, but unfortunately the surface of the wood was burned (high voltage $7 \mathrm{kV}$, duration 30 minutes, current with a closed shutter up to $260 \mu$ A) (Fig. 1). Samples for the TEM observation were prepared with ultramicrotome. Samples were embedded in epoxy resin and after setting they were cut to the shape of frustum pyramid. The upper surface of the frustum pyramid had a dimension of around $0.1 \mathrm{~mm}$. The samples were cut on an ultramicrotome machine which was fitted with a diamond knife. The diamond knife is equipped with a trough where the cut sections float on a water surface. The cut sections were collected from the water surface on the holey carbon copper grid.

In the case of SEM, we were looking for the best mode for observation in order to be able to examine the inner structure of wooden samples. For this purpose, we tested low vacuum mode and high vacuum mode with different coatings of cross-section (carbon, platinum). So far we achieved the best results with carbon coated samples observed under high vacuum (Fig. 2). It is important to remark that the quality of the carbon layer is highly dependent on the coating machine. We used comparable machines from different manufacturers with different results. SEM and HRSEM allowed us to have a general look on the wood cells and to a certain extent the cell walls. We obtained satisfactory information about the 
morphology. TEM examination gave us more information about the cell wall structure. It helped us compare cellulose nanocrystals from a reference sample with the UV-light irradiated samples.

\section{References:}

[1] M. Reza et al., "TEM wood fiber analysis review," BioResources 10(3) (2015) p. 6230-6261. [2] Plants 2018, 7(1), 14; https://doi.org/10.3390/plants7010014 (accessed November 17, 2018). [3] The authors acknowledge funding from the Institute of Physics, AS CR, v.v.i., Department of Material Analysis. This work is supported by the project GA18-26297S "Study of interactions in system: wooden surface - amorphus layer of $\mathrm{TiO} 2$ - crystalline layer $\mathrm{TiO} 2$ ”.).
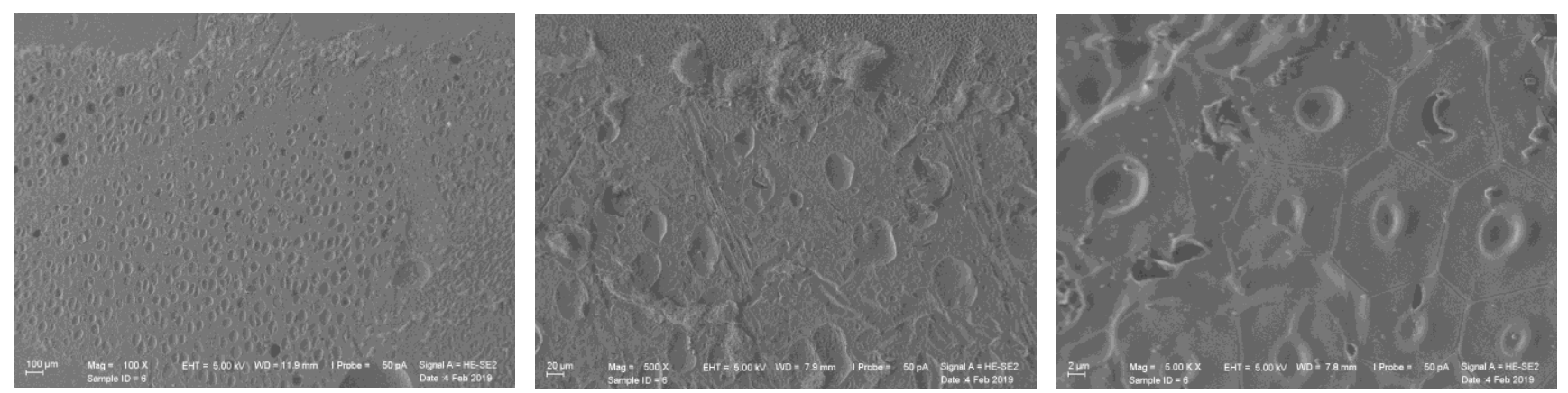

Figure 1. The beech wood polished and burned with PECS (HV $7 \mathrm{keV}$ ). Secondary electron image, magnification $100 \times, 500 \times, 5000 \times$ from left to right.
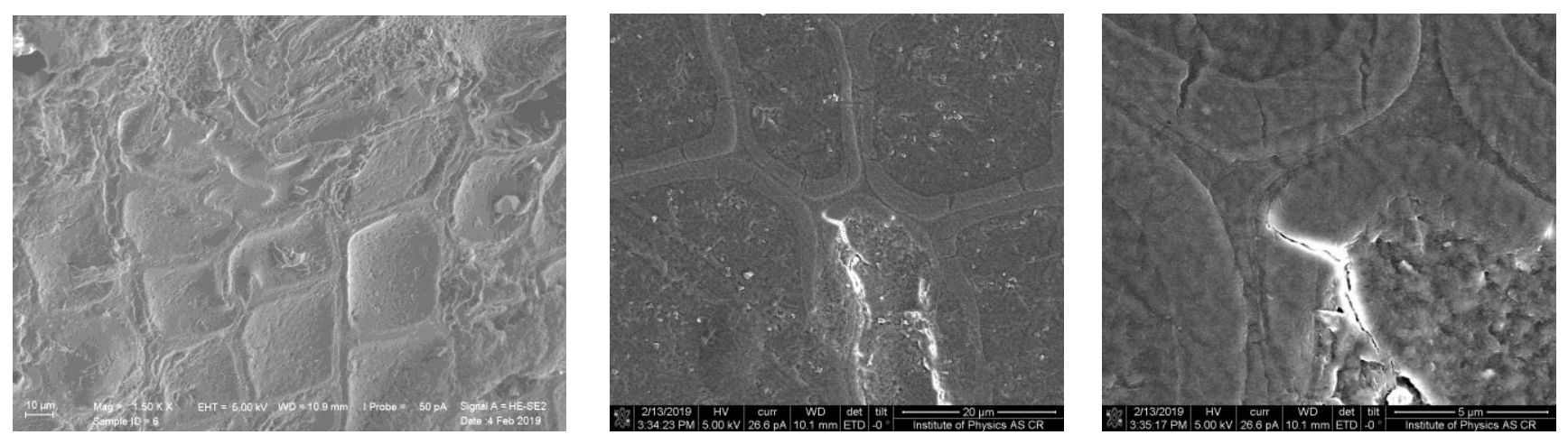

Figure 2. The pine wood coated with a carbon layer of $9.8 \mathrm{~nm}$. Wooden cells are close to the surface. The sample was not irradiated by UV light. Magnification 1500× (left). Wooden cells damaged by UV light. A general view on the wooden cells (in the middle) and details of wooden cell walls with cracks (right). Magnification $2000 \times$ and $8000 \times$ from the middle to the right. 